\title{
Arrested growth and spontaneous tumor regression of partially resected low-grade cerebellar astrocytomas in children
}

\author{
Joon-Khim Loh • Ann-Shung Lieu • Chee-Yin Chai • \\ Shiuh-Lin Hwang • Aij-Lie Kwan • Chih-Jen Wang • \\ Shen-Long Howng
}

Received: 31 March 2013 /Accepted: 11 April 2013 /Published online: 1 May 2013

(C) The Author(s) 2013. This article is published with open access at Springerlink.com

\begin{abstract}
Purpose The prognosis of children with low-grade cerebellar astrocytoma who have partial resection of tumor is largely unpredictable. The purpose of this study was to review the long-term outcome of such patients.

Methods The medical charts, imaging findings, operative notes, histopathological reports, and survival times of 12 patients with cerebellar astrocytoma were reviewed.

Results Five patients had total resection and seven had partial resection. Nine patients had grade I histology and three patients had grade II. Follow-up duration ranged from 3 to 25 years. Among the seven patients with residual tumor, five had tumor progression, one had arrested tumor growth, and one had spontaneous tumor regression. Five patients with partial resection received radiotherapy and three had malignant transformation of tumor during follow-up. Six patients, including five who had partial resection, underwent
\end{abstract}

J.-K. Loh • A.-S. Lieu • S.-L. Hwang • A.-L. Kwan • C.-J. Wang •

S.-L. Howng $(\bowtie)$

Division of Neurosurgery, Department of Surgery, Kaohsiung

Medical University Hospital, No 100 Tzyou 1 Rood,

Kaohsiung 807, Taiwan

e-mail: loststars111@gmail.com

J.-K. Loh • A.-S. Lieu • S.-L. Hwang • A.-L. Kwan • C.-J. Wang • S.-L. Howng

Faculty of Medicine, Graduate Institute of Medicine, College of Medicine, Kaohsiung Medical University, Kaohsiung, Taiwan

\section{J.-K. Loh}

Kaohsiung Municipal Hsiao-Kang Hospital, Kaohsiung Medical

University, Kaohsiung, Taiwan

C.-Y. Chai

Department of Pathology, Kaohsiung Medical University Hospital, Kaohsiung, Taiwan a second operation. One patient with partial resection died of pneumonia 23 years after surgery.

Conclusions Patients with complete tumor resection had a better prognosis than patients with partial resection. For patients with partial resection, we recommend a "wait and see" policy with surveillance using MRI. The phenomenon of arrested tumor growth and spontaneous tumor regression in patients with cerebellar astrocytoma who have subtotal resection warrants further study.

Keywords Cerebellar astrocytoma · Tumor regression · Magnetic resonance imaging $\cdot$ Radiation therapy

\section{Introduction}

Low-grade astrocytoma is the most common brain tumor in children [7]. About $40 \%$ of all tumors located in the posterior fossa are low-grade astrocytomas [7, 15, 17]. When these tumors are totally resected, the prognosis is very favorable with more than $90 \%$ of patients being cured without additional treatment [13]. The overall survival rate for children who had gross total resection of low-grade gliomas was reported to be $99 \%$ [16]. However, after incomplete resection, the prognosis is unpredictable $[5,10$, 13]. In many cases, the tumors will progress; however, in some cases, the tumor will have arrested growth or even tumor regression will occur $[5,10]$.

Relatively little attention has been paid in the literature in determining the incidence of arrested growth or spontaneous regression after partial resection of low-grade cerebellar astrocytomas and documenting such cases in detail. There is controversy about whether patients with tumor remaining after surgery should receive radiation therapy [5]. It is also 
unclear whether only patients with incomplete resection require follow-up and for how long [5].

Because of the lack of information on arrested growth and tumor regression after incomplete resection of lowgrade cerebellar astrocytomas in children, the aim of this study was to provide more information on these phenomena by reviewing cases of patients with cerebellar astrocytoma who underwent surgery at our hospital.

\section{Patients and methods}

We reviewed all cases of patients under 16 years of age who were diagnosed with cerebellar astrocytoma and underwent surgery at Kaohsiung Medical University Hospital from January 1983 to December 2011. Medical charts, imaging findings, operative notes, histopathological reports, and survival times of 12 patients with cerebellar astrocytoma were reviewed for the purpose of evaluating the patients' prognosis; patients lost to follow-up were excluded. This study was approved by our internal review board

\section{Results}

\section{Patient characteristics}

The clinical data for the 12 patients in this study are summarized in Table 1. Six patients were boys and six were girls. Their age at the time of diagnosis ranged from 3 to 16 years. Nine patients $(75 \%)$ presented with gait disturbance, eight $(66.7 \%)$ with headache, six $(50 \%)$ with vomiting, and four $(33.3 \%)$ with a visual problem. The tumor was located in the midline in seven patients $(58.3 \%)$, in the left cerebellum in three patients $(25 \%)$ and in the right cerebellum in two patients $(16.7 \%)$.

\section{Surgical data}

The tumor was totally removed in five patients $(41.7 \%)$ whereas seven patients $(58.3 \%)$ had only partial tumor removal. There were no signs of type 1 neurofibromatosis such as neurofibromas or café au lait spots over the body. Histological examination of surgical specimens showed that nine tumors were classic pilocytic astrocytoma (grade I) and three were diffuse (grade II) astrocytoma.

\section{Postoperative follow-up}

Follow-up ranged from 3 to 25 years. One patient who had total resection (case 4) and five patients who had partial resection (cases 1, 2, 3, 6, and 10) underwent a second surgical operation. In addition, three patients with subtotal resection, all of whom had the tumor located in the midline, received a ventriculoperitoneal shunt. Among the seven patients who had incomplete tumor resection in the initial operation, five patients $(71.4 \%)$ had tumor progression detected during follow-up and two patients $(28.6 \%)$ did not have any tumor progression detected during follow-up of 17 years (case 6) and 11 years (case 9). The time at which progression occurred is indicated by time of reoperation in Table 1. In one of these two patients who did not have tumor progression (case 9), serial T1 MRI with gadolinium demonstrated tumor regression from February 2008 to January 2011 with the tumor getting smaller (Fig. 1). Among the five patients who had total resection, one patient (case 5) with diffuse astrocytoma (grade II) received radiation therapy. Five of the patients with subtotal resection were treated with radiation therapy, which is still used in some hospitals for patients with subtotal resection of cerebellar low-grade astrocytomas. Post-irradiation follow-up of the patients irradiated for residual tumor revealed that in one patient (case 1) there was malignant transformation from grade I to grade II at 2 years after surgery and in another patient (case 2) there was malignant transformation from grade I to grade III (anaplastic astrocytoma) at 15 years after surgery. One of the patients (case 3) with partial tumor resection who was treated with radiation therapy developed radiation-induced anaplastic meningioma over the supratenorium after 23 years of follow-up (Fig. 2a). The meningioma was completely removed (Fig. 2b).

Among the 12 patients, 11 were alive at the end of the study. One patient (case 1) died of pneumonia 23 years after undergoing surgery.

\section{Discussion}

In this study, we analyzed the long-term follow-up results of 12 patients with childhood cerebellar astrocytoma: five of whom had total tumor resection and seven of whom had subtotal tumor resection. As expected, total tumor removal was an important prognostic factor. Five of the seven patients with subtotal resection had tumor progression. However, two patients with subtotal resection did not have tumor progression and one of these patients had spontaneous tumor regression which was well documented on serial MRI. Half of the patients, including five with subtotal resection were treated with radiation therapy. Three of these patients had malignant transformation of tumor.

We found that complete resection of cerebellar astrocytoma is an important prognostic factor, indicating a more favorable prognosis than subtotal resection. This was also the conclusion of a much larger study by Villarejo et al. [15] who reviewed 203 cases of low-grade cerebellar astrocytoma. They also found that that location, size, and histology of 


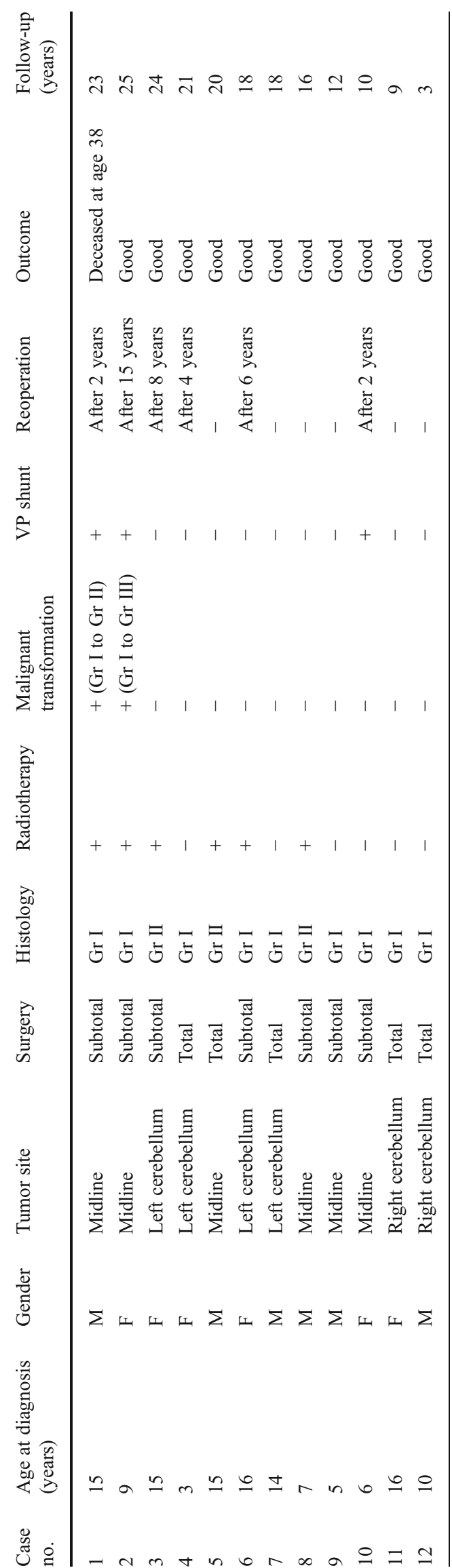

tumors have a significant effect on prognosis; the tumors located in one hemisphere, that are small in size, and are either cystic or have a cystic posterior nodule have a better prognosis. However, Beebe et al. [3] in a study of 103 children with low-grade cerebellar astrocytomas found that cognitive and adaptive outcome was not related to tumor location. In a prospective study of 518 children with lowgrade glioma, Wisoff et al. achieved gross total resection of the tumor in $64 \%$ of the patients. They found that the only factor that was highly predictive of progression-free survival in multivariate analysis was gross total resection; location, histological type, and age were not significant independent predictors of progression-free survival [16].

A number of studies have looked at various measurements of outcomes in patients with cerebellar astrocytomas, although without considering whether tumors were completely resected or not during surgery. In a study that looked at not only neurological outcomes of surgery but also behavioral and emotional adjustment and health-related quality of life (HRQoL) in children with low-grade cerebellar astrocytomas, it was found that although $43 \%$ of patients had neurological sequelae, $19 \%$ had cognitive deficits that caused significant problems in schools, and $27 \%$ had emotional adjustment disturbances, the patients rated their HRQoL as normal or in some instances actually higher than healthy controls [18]. In contrast, it has been reported that in patients treated for low-grade astrocytoma, QoL was significantly decreased in every domain except for emotions [2]. It has also been found that in patients with cerebellar astrocytoma who were treated surgically, during follow-up ranging from 1 year to almost 9 years, numerous deficits in various combinations and different degrees were detected including apraxia, motor neglect, and dysarthric characteristics plus other deficits such as language, memory, and behavioral problems [1]. The authors noted that whereas cerebellar pilocytic astrocytoma is generally considered to be a benign tumor that is associated with a good long-term quality of life, they found that following surgical treatment it results in long-term neurocognitive disturbances resulting in a high percentage of children needing special education.

The role of radiation therapy in treating children with tumors is controversial. Galloway et al. [6] studied 370 consecutive patients with solid tumors or leukemia who were treated with curative radiotherapy. They found that 18 second tumors occurred in 16 patients and, that during long-term follow-up, the risk for second tumor does not plateau. The majority of second tumors were meningiomas that could be successfully removed. The authors concluded that despite the risk of a second tumor the primary threat to survival is failure to control the primary tumor and therefore radiotherapy should be used selectively. In our study, six patients received radiotherapy and two cases (cases 1 and 2) had malignant transformation of tumor, and another (case 3) 

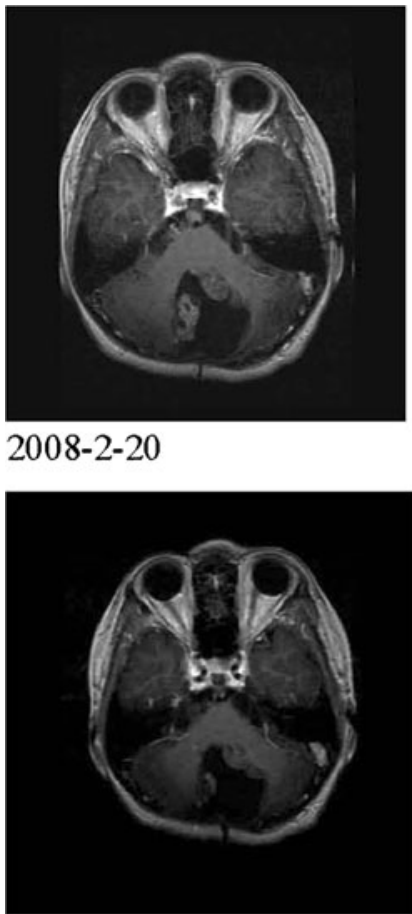

2009-12-30
2008-2-20

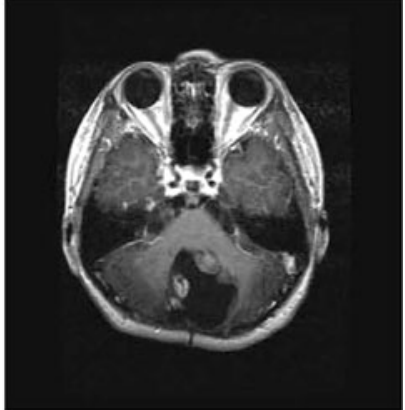

$2009-1-22$

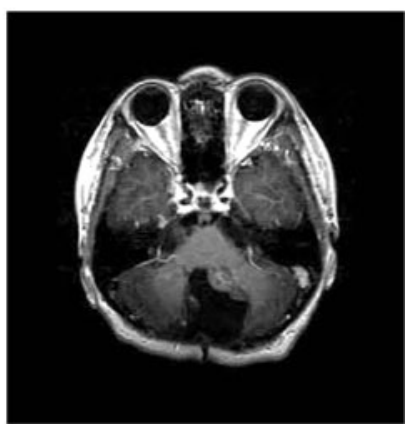

$2011-1-24$
Fig. 1 Serial brain MRI scans of case 9 show regression of the cerebellar astrocytoma

had radiation-induced anaplastic meningioma develop over the supratenorium. Radiation-induced meningiomas have been reported in the literature $[4,9]$. We do not recommend radiation therapy for partial resection of childhood cerebellar astrocytoma because it has not been demonstrated in most studies to reduce the rate of recurrence or prolong overall survival [10]. It should be noted, however, that Hadjipanavis et al. [8] found radiotherapy to be useful for treating patients with recurrent or subtotal resection of pilocytic astrocytoma. We also do not recommend radiotherapy because cerebellar astrocytomas are insensitive to radiation therapy and there is a risk of radiation-induced necrosis as well as second malignancy. The rate of malignant transformation was very high in our study $(33.3 \%)$,
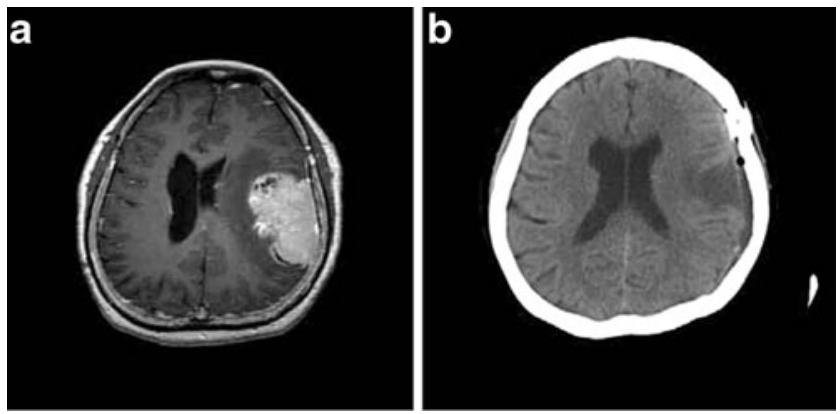

Fig. 2 a MRI scan of case 3 reveals anaplastic meningioma over the supratenorium left parietal lobe. b Brain CT scan reveals total removal of the meningioma especially considering that such transformation is considered to be a rare event [6]. We are unable to explain this high rate. One possibility is a biopsy sampling error 2 years after surgery. For patients who have tumor progression, surgery for tumor removal may be recommended.

Our study showed that after partial excision a small proportion of cerebellar astrocytomas do not progress or even regress. Among the seven patients in our study with partial resection, in one there was arrested growth and in another there was regression; the other five patients had progression. These phenomena of arrested growth and regression have been reported in a number of studies [5, 7, 11-14]. Ogiwara et al. [10] found that among 50 residual cerebellar astrocytomas, $16 \%$ demonstrated arrested growth and $32 \%$ demonstrated spontaneous regression. As have a number of other authors, we recommend a "wait and see" policy for patients with partial resection in which the patients are followed up with MRI $[5,7,11,12]$.

The mechanism of spontaneous regression of cerebellar astrocytoma is unknown. Among the various mechanisms suggested have been immunologic mechanisms, induction of differentiation, and elimination of a carcinogen, ischemic tumor necrosis, and apoptosis [13]. Ogiwara et al. [10] found that the mean time for tumor regression in their series was 13.6 months which led them to suggest that the mechanism involved ischemic necrosis and/or apoptosis. Steinbok et al. [13] showed that an apoptosis marker, ApoPtag, was positive within the cerebellar astrocytoma of a patient with tumor regression, which was an indication that the mechanism of regression may have involved the apoptosis-related genes.

Our study had several limitations. It was retrospective in design. The sample size was small and it was conducted at one center. Assessment of long-term outcome did not include evaluation of cognitive function, emotional disturbances, behavior disorders, educational problems, or HRQoL.

\section{Conclusion}

We have documented that patients with subtotal removal of cerebellar astrocytoma can have arrested tumor growth or spontaneous tumor regression during long-term follow-up. Following partial resection of pediatric cerebellar astrocytoma, we recommend that the patients be followed up a "wait and see" approach with surveillance using MRI. We found that several tumors treated with radiotherapy after surgery had malignant transformation and do not recommend adjuvant radiation treatment for children with cerebellar astrocytoma who have subtotal resection. More research is needed on the prognosis of patients with subtotal resection of cerebellar astrocytoma. 
Conflicts of interest None to declare.

Open Access This article is distributed under the terms of the Creative Commons Attribution License which permits any use, distribution, and reproduction in any medium, provided the original author(s) and the source are credited.

\section{References}

1. Aarsen FK, Van Dongen HR, Paquier PF, Van Mourik M, Catsmann-Berrevoets C (2004) Long-term sequelae in children after cerebellar astrocytoma surgery. Neurology 62:1311-1316

2. Aarsen FK, Paquier PF, Reddingius RE, Streng IC, Arts W-FM, Evera-Preesman M (2006) Functional outcome after low-grade astrocytoma treatment in childhood. Cancer 106:396-402

3. Beebe DW, Ris MD, Armstrong D, Fontanesi J, Mulhern R, Holmes E, Wisoff JH (2005) Cognitive and adaptive outcome in low-grade pediatric cerebellar astrocytomas: evidence of diminished cognitive and adaptive functioning in national collaborative research studies (CCG 989/POG 9130). J Clin Oncol 23:5198-204

4. Chowdhary A, Spence AM, Sales L, Rostomily RC, Rockhill JK, Silbergeld DL (2012) Radiation associated tumors following therapeutic cranial radiation. Surg Neurol Int 3:48. doi:10.4103/21527806.96068

5. Dirven CM, Mooij JJ, Molenaar WM (1997) Cerebellar pilocytic astrocytoma: a treatment protocol based upon analysis of 73 cases and a review of the literature. Childs Nerv Syst 13:17-23

6. Galloway TJ, Indelicato DJ, Amdur RJ, Swanson EL, Smith AA, Marcus RB Jr (2012) Second tumors in pediatric patients treated with radiotherapy to the central nervous system. Am J Clin Oncol 35:279-283

7. Gunny RS, Hayward RD, Phipps KP, Harding BN, Saunders DE (2005) Spontaneous regression of residual low-grade cerebellar pilocytic astrocytomas in children. Pediatr Radiol 35: 1086-1091
8. Hadjipanavis CG, Kondiolka D, Gardner P, Niranjan A, Dagam S, Flickinger JC, Lunsford LD (2002) Stereotactic radiosurgery for pilocytic astrocytomas when multimodal therapy is necessary. $\mathrm{J}$ Neurosurg 97:56-64

9. Kamashima Y, Terasaka S, Kobayashi H, Kaneko S, Kubota K, Tanaka S, Houkin K (2012) Radiation induced intraparenchymal meningioma occurring 6 years after CNS germinoma: case report. Clin Neurol Neurosurg 114:1077-1080

10. Ogiwara H, Bowman RM, Tomita T (2012) Long-term follow-up of pediatric benign cerebellar astrocytoma. Neurosurgery 70:40-48

11. Palma L, Celli P, Mariottini A (2004) Long-term follow-up of childhood cerebellar astrocytomas after incomplete resection with particular referenced to arrested growth or spontaneous tumour regression. Acta Neurochir (Wein) 146:581-588

12. Saunders DE, Phipps KP, Hons KP, Wade AM, Hayward RD (2005) Surveillance imaging strategies following surgery and or/ radiotherapy for childhood cerebellar low-grade astrocytoma. J Neurosurg 102((2 Suppl)):172-178

13. Steinbok P, Poskitt K, Hendson G (2006) Spontaneous regression of cerebellar astrocytoma after subtotal resection. Childs Nerv Syst 226:572-576

14. Vassilyadi M, Shamji MF, Tataryn Z, Keene D, Ventureyra (2009) Postoperative surveillance magnetic resonance imaging for cerebellar astrocytoma. Can J Neurol Sci 36:707-712

15. Villarejo F, Diego JMB, Riva AG (2008) Prognosis of cerebellar astrocytoma in children. Childs Nerv Syst 24:203-210

16. Wisoff JH, Sanford RA, Heier LA, Sposto R, Burger PC, Yates AJ, Holmes EJ, Kun LE (2011) Primary neurosurgery for pediatric low-grade gliomas: a prospective multi-institutional study from the Children's Oncology Group. Neurosurgery 68:15481555

17. Youmans JR (ed) (1990) Neurological surgery, 3rd edn. W.B. Saunders, Philadelphia

18. Zuzak TJ, Poretti A, Drexel B, Zehnder D, Boltshauser E, Grotzer MA (2008) Outcome of children with low-grade cerebellar astrocytoma: long-term complications and quality of life. Childs Nerv Syst 24:1447-55 